\title{
Substructural Fuzzy-Relevance Logic
}

\author{
Eunsuk Yang
}

\begin{abstract}
This paper proposes a new topic in substructural logic for use in research joining the fields of relevance and fuzzy logics. For this, we consider old and new relevance principles. We first introduce fuzzy systems satisfying an old relevance principle, that is, Dunn's weak relevance principle. We present ways to obtain relevant companions of the weakening-free uninorm (based) systems introduced by Metcalfe and Montagna and fuzzy companions of the system $\mathbf{R}$ of relevant implication (without distributivity) and its neighbors. The algebraic structures corresponding to the systems are then defined, and completeness results are provided. We next consider fuzzy systems satisfying new relevance principles introduced by Yang. We show that the weakening-free uninorm (based) systems and some extensions and neighbors of $\mathbf{R}$ satisfy the new relevance principles.
\end{abstract}

\section{Introduction}

The purpose of this paper is to extend the world of fuzzy logic to the realm of relevance logic, and vice versa. For this purpose, recall first some historical facts associated with fuzzy and relevance logics. Fuzzy logic based on t-norms has a distinguished history, the most famous examples being $\mathbf{L}$ (Lukasiewicz logic), $\mathbf{G}$ (GödelDummett logic), $\prod$ (product logic), BL (basic fuzzy logic), and MTL (monoidal t-norm logic). These logic types are generally called t-norm (based) logic. T-norm logic is not a type of relevance logic because, while such logic proves the weakening (W) $\varphi \rightarrow(\psi \rightarrow \varphi)$, an arbitrary logic with (W) and modus ponens admits of a theorem $\varphi \rightarrow \psi$ such that $\varphi$ and $\psi$ are irrelevant to each other. The system $\mathbf{R M}$ (the $\mathbf{R}$ of relevant implication with mingle) has been considered as a type of relevance logic. In particular, Dunn [7] investigated RM capturing the tautologies on denumerable infinite sets of truth values and showed that $\mathbf{R M}$ is complete with respect to (w.r.t.) linearly ordered Sugihara matrices. According to Cintula [5], a (weakly implicative)

Received December 11, 2010; accepted May 13, 2013

2010 Mathematics Subject Classification: Primary 02C; Secondary 02J

Keywords: (substructural) fuzzy-relevance logic, fuzzy logic, relevance logic, uninorm (based) logic

(C) 2015 by University of Notre Dame 10.1215/00294527-3132824 
$\operatorname{logic} \mathbf{L}$ is said to be fuzzy if it is complete w.r.t. linearly ordered matrices (or algebras). Then, though RM is not a t-norm logic, it is still a fuzzy logic in Cintula's sense and is thus a type of fuzzy and relevance logic.

One interesting point is that Sugihara algebra with identity on the real unit interval $[0,1]$ is a uninorm, a generalization of t-norms where the identity can lie anywhere in $[0,1]$ (see Yager and Rybalov [21]). Metcalfe [15] introduced the uninorm logic $\mathbf{U L}$, which captures the tautologies of left-continuous conjunctive uninorms and their residua, as a weakening of MTL and a strengthening of MAILL (multiplicative additive intuitionistic linear logic). Recently, Metcalfe and Montagna [16] have investigated UL and several axiomatic extensions of it as substructural fuzzy logical systems lacking structural rules like weakening or contraction. They introduced the weakening-free uninorm systems UL, IUL (involutive uninorm logic), UML (uninorm mingle logic), and IUML (involutive uninorm mingle logic). Among them, IUML is the $\mathbf{R M}^{\mathbf{T}}$ (RM plus constants $\mathbf{T}, \mathbf{F}$ and the corresponding axioms) with (FP) $\mathbf{t} \leftrightarrow \mathbf{f}$ (see Definition 14 below). ${ }^{1}$ Thus, the system IUML may be regarded as a uninorm (based) version of $\mathbf{R M}$ or $\mathbf{R M}^{\mathbf{T}}$ in the sense that the logic characterized by models based on $[0,1]$ is IUML but not $\mathbf{R} \mathbf{M}^{(\mathbf{T})}{ }^{2}$ Furthermore, since the system UL is a weakening of $\mathbf{R} \mathbf{M}^{\mathbf{T}}$, both $\mathbf{R} \mathbf{M}^{\mathbf{T}}$ and $\mathbf{U L}$ seem to be not merely fuzzy, but relevant. (Note that relevance systems such as $\mathbf{R}, \mathbf{R M}, \mathbf{E}$ of entailment and $\mathbf{T}$ of ticket entailment all reject (W).) Therefore, it makes sense to wonder if the weakening-free uninorm systems introduced in [16] are all relevant.

The answer depends on the circumstances. Under the following situation, the answer is "no." We most frequently call a system relevant if it satisfies the strong relevance principle (SRP) in Anderson and Belnap [1] that $\varphi \rightarrow \psi$ is a theorem only if $\varphi$ and $\psi$ share a propositional variable, and sometimes if it satisfies the weak relevance principle (WRP) in Dunn [7] that $\varphi \rightarrow \psi$ is a theorem only if either (i) $\varphi$ and $\psi$ share a propositional variable, or (ii) both $\neg \varphi$ and $\psi$ are theorems. For instance, the system $\mathbf{R}$ is strongly relevant in that it satisfies the principle SRP, and the system RM is weakly relevant in that it satisfies the principle WRP. However, the system $\mathbf{U L}$ is neither strongly nor weakly relevant because it proves such formulas as $(\alpha)(\varphi \wedge \neg \varphi) \rightarrow(\psi \vee \neg \psi)$. Instead, although proving $(\alpha)$, the system IUML seems to be weakly relevant because it proves (EM) $\varphi \vee \neg \varphi$, and so the statements $\neg(\varphi \wedge \neg \varphi)$ and $\psi \vee \neg \psi$ are both theorems of IUML. However, since IUML also proves $(\beta)((\varphi \rightarrow \mathbf{F}) \wedge \varphi) \rightarrow \psi$, it still does not satisfy WRP. Therefore, none of the weakening-free uninorm systems are relevant in the sense that they satisfy neither SRP nor WRP.

Next, consider the circumstances under which the answer is "yes." Very recently, the present author [22] introduced new strong and weak relevance principles because the principles SRP and WRP do not work on relevance systems with propositional constants (see Galatos, Jipsen, Kowalski, and Ono [11] and Restall [20]). According to him, a system is said to be strongly relevant if it satisfies the new strong relevance principle (NSRP) in [22] that $\varphi \rightarrow \psi$ is a theorem only if $\varphi$ and $\psi$ either explicitly or strong implicitly share a propositional variable, and weakly relevant if it satisfies the new weak relevance principle (NWRP) that $\varphi \rightarrow \psi$ is a theorem only if either (i) $\varphi$ and $\psi$ share either explicitly or strong implicitly share a propositional variable, or (ii) both $\neg \varphi$ and $\psi$ are theorems (see Section 3 below). ${ }^{3}$ The weakening-free systems in [16] are all relevant in the sense that they satisfy the principle NSRP or the principle NWRP since theorems such as $(\alpha)$ and $(\beta)$ strong implicitly share at 
least one propositional variable. Therefore, they are all relevant in that they satisfy NSRP or NWRP.

Let us call the relevance principles in [1] and [7] old relevance principles, and the relevance principles in [22] new relevance principles. Here, we introduce logics being both fuzzy in Cintula's sense and relevant in the old and new senses.

Related to the old relevance principles, one interesting point is that, while IUML proves the sentence $(\beta)$, the IUML without constants $\mathbf{T}, \mathbf{F}$ and the corresponding axioms (RIUML) does not. This shows that, among the systems obtained from the weakening-free uninorm systems introduced in [16] by omitting constants $\mathbf{T}, \mathbf{F}$ and the corresponding axioms, systems proving (EM) satisfy WRP and so are weakly relevant in the old sense (see Theorem 2 in Section 2). This provides a way to obtain relevant companions of the weakening-free uninorm systems in [16], that is, the method that drop constants $\boldsymbol{T}, \boldsymbol{F}$ (and the corresponding axioms) from a weakeningfree uninorm logic $\boldsymbol{L}$, but instead add $(E M)$ to $\boldsymbol{L}$ if it does not prove it. Using this method, we can obtain weakening-free fuzzy systems satisfying WRP and so weakly (but not strongly) relevant in the old sense. We verify this by introducing several systems. More exactly, in Section 2.1, we first introduce the relevant uninorm logic RUL, the logic for a relevant companion of the uninorm logic $\mathbf{U L}$, and its axiomatic extensions (i.e., relevant companions of IUL, UML, and IUML) as substructural relevant fuzzy logics. We can analogously consider fuzzy companions of relevance systems by adding an axiom ensuring prelinearity (together with (EM)) to relevance systems. Next, we introduce a method to obtain fuzzy companions of the relevance systems $\mathbf{R W}$ (the $\mathbf{R}$ without contraction), $\mathbf{R}, \mathbf{R M}$, or of their distributivity-free systems LRW, LR, and LRM, respectively. More precisely, we next introduce the contractionless fuzzy relevant logic FRW, the logic for a fuzzy companion of both LRW and RW (briefly (L)RW), and its axiomatic extensions FR, FRM (i.e., fuzzy companions of (L)R, (L)RM) as substructural fuzzy relevant logics. We will call these two types of logic substructural fuzzy-relevance logics. The results will show that the fuzzy relevant logics are also relevant fuzzy logics because the systems are between the weakest relevant fuzzy logic RUL and the strongest logic RIUML.

Many logics with the "prelinearity" axiom $\left(\mathrm{PL}_{\mathbf{t}}\right)(\mathrm{A} 11$ in Definition 2 below) are complete w.r.t. linearly ordered algebras (or matrices). ${ }^{4}$ For example, the system UL is obtained by adding $\left(\mathrm{PL}_{\mathbf{t}}\right)$ to MAILL and complete w.r.t. linearly ordered UL-algebras. In Section 2.2, we define algebraic structures corresponding to the systems introduced in Section 2.1 and then prove their completeness. In fact, since the method of the algebraic completeness proof is standard, we will instead show that they are weakly implicative fuzzy logics, the class of which is presented in [5]. This implies that they are all fuzzy logics in Cintula's sense. Furthermore, we prove that they also satisfy WRP. This will ensure that they are all weakly relevant in the old sense and so both fuzzy and relevant. Therefore, the study will introduce, in the view of substructural logic, one new research area-fuzzy-relevance logic—bridging fuzzy logic and relevance logic, each of which has been independently investigated. (Namely, it introduces the logics belonging to the intersection of the families of fuzzy and relevance logics.)

In addition, in Section 2.3, we briefly consider the fuzzy-relevance systems eliminating $\left(\mathrm{PL}_{\mathbf{t}}\right)$ as both weakly implicative logics, the class of which is also presented in [5], and substructural relevance logics. 
The fuzzy-relevance systems introduced in Section 2 would not be interesting to some (or many) fuzzy logicians because they are not t-norm systems nor even uninorm (based) systems. Instead, the principles NSRP and NWRP may be interesting to such logicians because these principles ensure that some weakening-free uninorm systems are relevant. More precisely, in Section 3, we preliminarily introduce NSRP and NWRP because they are not familiar to the readers. In Section 4.1, we prove that the weakening-free uninorm systems in [16] and the fuzzy companions of the relevance systems with constants $\mathbf{T}$ and $\mathbf{F} \mathbf{F R W}^{\mathbf{T}}, \mathbf{F R}^{\mathbf{T}}, \mathbf{F R M}^{\mathbf{T}}$ all satisfy NSRP or NWRP. In Section 4.2, we introduce a method to obtain strong relevant companions from UL and its weakening-free extensions. We also call the systems introduced in Sections 4.1 and 4.2 substructural fuzzy-relevance logics. In addition, as in Section 2.3, in Section 4.3, we consider substructural relevance logics obtained from the systems by omitting $\left(\mathrm{PL}_{\mathbf{t}}\right)$.

All the systems (i.e., the systems with and without $\left(\mathrm{PL}_{\mathbf{t}}\right)$ ) introduced in Sections 2 and 4 are substructural logics placed somewhere over $\mathbf{F L}_{e}$ (full Lambek logic with exchange) (see Remark 3 below). Thus, all the (fuzzy-)relevance logics investigated here are substructural logics. For simplicity, we henceforth call them (fuzzy-)relevance logics and not substructural (fuzzy-)relevance logics.

For convenience, we adopt the notation and terminology similar to that in Cintula [5], Esteva and Godo [9], [10], Hájek [12], and Metcalf and Montagna [16] and assume familiarity with them (together with the results found therein).

\section{Fuzzy-Relevance Logics (I)}

In this section, we introduce several fuzzy-relevance systems satisfying the principle WRP and their corresponding non-fuzzy-relevance systems.

2.1 Syntax We base (fuzzy-)relevance logics on a countable propositional language with formulas FOR built inductively as usual from a set of propositional variables $V A R$, binary connectives $\rightarrow, \&, \wedge, \vee$, and constants $\mathbf{f}, \mathbf{t}$, with defined connectives:

df1. $\neg \varphi:=\varphi \rightarrow \mathbf{f}$, and

df2. $\varphi \leftrightarrow \psi:=(\varphi \rightarrow \psi) \wedge(\psi \rightarrow \varphi)$.

We moreover define $\varphi_{\mathbf{t}}^{n}$ as $\varphi_{\mathbf{t}} \& \cdots \& \varphi_{\mathbf{t}}, n$ factors, where $\varphi_{\mathbf{t}}:=\varphi \wedge \mathbf{t}$, and similarly for $\varphi^{n}$.

For the remainder of the paper, we will utilize the customary notation and terminology. We use the axiom systems to provide a consequence relation.

We start with the following axiomatization of RMAILL (relevant multiplicative additive intuitionistic linear logic) as the basic relevance logic defined here. ${ }^{5}$

Definition 1 RMAILL consists of the following axiom schemes and rules:

A1. $\varphi \rightarrow \varphi$

A2. $(\varphi \wedge \psi) \rightarrow \varphi,(\varphi \wedge \psi) \rightarrow \psi$

A3. $((\varphi \rightarrow \psi) \wedge(\varphi \rightarrow \chi)) \rightarrow(\varphi \rightarrow(\psi \wedge \chi))$

A4. $\varphi \rightarrow(\varphi \vee \psi), \psi \rightarrow(\varphi \vee \psi)$

A5. $((\varphi \rightarrow \chi) \wedge(\psi \rightarrow \chi)) \rightarrow((\varphi \vee \psi) \rightarrow \chi)$

A6. $(\varphi \& \psi) \rightarrow(\psi \& \varphi)$

A7. $(\varphi \& \mathbf{t}) \leftrightarrow \varphi$

A8. $(\varphi \rightarrow(\psi \rightarrow \chi)) \leftrightarrow((\varphi \& \psi) \rightarrow \chi)$

A9. $(\varphi \rightarrow \psi) \rightarrow((\psi \rightarrow \chi) \rightarrow(\varphi \rightarrow \chi))$ (self-implication, SI)

$(\wedge$-elimination, $\wedge$-E)

$(\wedge$-introduction, $\wedge-\mathrm{I})$

$(\vee$-introduction, $\vee-\mathrm{I})$

$(\vee$-elimination, $\vee$-E)

(\&-commutativity, \&-C)

(push and pop, PP)

(residuation, RE)

(suffixing, SF) 


$$
\begin{aligned}
& \text { A10. } \varphi \vee \neg \varphi \\
& \varphi \rightarrow \psi, \varphi \vdash \psi \\
& \varphi, \psi \vdash \varphi \wedge \psi
\end{aligned}
$$$$
\text { (excluded middle, EM) }
$$

Relevant uninorm logic RUL, the basic relevant fuzzy logic defined here, is RMAILL extended with the "prelinearity" axiom scheme below.

Definition 2 RUL is RMAILL plus

$$
\text { A11. }(\varphi \rightarrow \psi)_{\mathbf{t}} \vee(\psi \rightarrow \varphi)_{\mathbf{t}}
$$

Relevant fuzzy logics are defined by extending RUL with suitable axiom schemes as follows.

Definition 3 A logic is an axiomatic extension (extension for short) of $\mathbf{L}$ if and only if (iff) it results from the addition of axiom scheme(s) to L. In particular, the following are relevant fuzzy logics extending RUL:

- involutive RUL RIUL is RUL plus (DNE) $\neg \neg \varphi \rightarrow \varphi$;

- idempotent RUL RUML is RUL plus (ID) $(\varphi \& \varphi) \leftrightarrow \varphi$;

- involutive RUML RIUML is RIUL plus (ID) and (FP) $\mathbf{t} \leftrightarrow \mathbf{f}$.

Remark 1 By eliminating the axiom (EM) from the systems RUL, RIUL, RUML, and RIUML and adding propositional constants T, F (and the corresponding axioms, i.e., A12 and A13 in Section 4), we obtain the weakening-free uninorm systems UL, IUL, UML, and IUML, respectively, as introduced in [16]. However, note that, since IUML proves (EM), we obtain IUML simply by adding constants T, F (and the corresponding axioms) to RIUML.

The system $\mathbf{L R W}$ is the $\mathbf{F L}_{e}$ with (DNE). Fuzzy relevant logics are defined by extending LRW or RMAILL with suitable axiom schemes as follows.

Definition 4 The following are fuzzy relevant logics extending LRW:

- fuzzy LRW FRW is LRW plus A11 and (EM);

- fuzzy LR FR is FRW plus (SIN) $\varphi \rightarrow(\varphi \& \varphi)$;

- fuzzy LRM FRM (= RM) is FR plus (SDE) $(\varphi \& \varphi) \rightarrow \varphi$.

We may instead consider the systems FRW, FR, and FRM as fuzzy relevant logics extending RW. (Note that the system RW is LRW plus (distributivity, D) $(\varphi \wedge(\psi \vee \chi)) \rightarrow((\varphi \wedge \psi) \vee(\varphi \wedge \chi))$, and so we can obtain FRW, FR, and FRM by adding the axiom scheme(s) in Definition 4 to the systems RW, R, and $\mathbf{R M}$, respectively.) However, here we introduce them as extensions of $\mathbf{L R W}$ in place of RW because the basic relevance logic RMAILL is a distributivity-free system.

Remark 2 By eliminating both A11 and (EM) from the system FRW, we obtain the distributivity-free relevance logic LRW; by eliminating A11 from the systems FR and FRM, we get LR and LRM, respectively. Note that LR and LRM each prove (EM). Note also that the systems FRW and FRM are the same as RIUL and $\mathbf{R M}$, respectively, and that the system RIUML is FRM (= RM) plus (FP). Therefore, the fuzzy relevant logics FRW, FR, and RM are all between RUL and RIUML and so are relevant fuzzy logics extending RUL. Tables 1 and 2 summarize some axiom schemes and the extensions of RMAILL introduced above.

For easy reference, we let Ls be the set of fuzzy-relevance logics defined previously. 
Table 1 Some axiom schemes in fuzzy-relevance logics.

\begin{tabular}{|l|l|}
\hline Axiom schema & Name \\
\hline$\neg \neg A \rightarrow A$ & Double negation elimination (DNE) \\
\hline$A \rightarrow A \& A$ & Square increasing (SIN) \\
\hline$A \& A \leftrightarrow A$ & Idempotence (ID) \\
\hline $\mathbf{t} \leftrightarrow \mathbf{f}$ & Fixed-point (FP) \\
\hline$(\varphi \rightarrow \psi)_{\mathbf{t}} \vee(\psi \rightarrow \varphi)_{\mathbf{t}}$ & Prelinearity (PL $\mathbf{t})$ \\
\hline
\end{tabular}

Table 2 Some extensions of RMAILL obtained by adding the corresponding additional axiom schemes.

\begin{tabular}{|l|l|}
\hline Logic & Additional axiom schemes \\
\hline RUL & $\left(\mathrm{PL}_{\mathfrak{t}}\right)$ \\
\hline RIUL $(=$ FRW $)$ & $\left(\mathrm{PL}_{\mathfrak{t}}\right)$ and $(\mathrm{DNE})$ \\
\hline FR & $\left(\mathrm{PL}_{\mathfrak{t}}\right),(\mathrm{DNE})$ and $(\mathrm{SIN})$ \\
\hline RUML & $\left(\mathrm{PL}_{\mathfrak{t}}\right)$ and $(\mathrm{ID})$ \\
\hline RM $(=$ FRM $)$ & $\left(\mathrm{PL}_{\mathfrak{t}}\right),(\mathrm{DNE})$ and $(\mathrm{ID})$ \\
\hline RIUML & $\left(\mathrm{PL}_{\mathfrak{t}}\right),(\mathrm{DNE}),(\mathrm{ID})$ and $(\mathrm{FP})$ \\
\hline
\end{tabular}

Definition 5 Ls $=\{$ RUL, RIUL (= FRW), RUML, RIUML, FR, RM $(=\mathbf{F R M})\}$.

A theory is a set of formulas. A proof in a theory $T$ over $\mathrm{L}(\in \mathrm{Ls})$ is a sequence $s$ of formulas such that each element of $s$ is either an axiom of $\mathrm{L}$, a member of $T$, or is derivable from previous elements of $s$ by means of a rule of L. $T \vdash \varphi$, more exactly $T \vdash_{L} \varphi$, means that $\varphi$ is provable in $T$ w.r.t. L, that is, there is an L-proof of $\varphi$ in $T$. If $\vdash_{L} \varphi$, that is, $T=\emptyset, \varphi$ is said to be a theorem of L. A theory $T$ is said to be inconsistent if $T \vdash \mathbf{F}$; otherwise it is consistent.

The relevant (local) deduction theorem $(\mathrm{R}(\mathrm{L}) \mathrm{DT})$ for $\mathrm{L}$ is as follows.

Proposition $1 \quad$ Let $T$ be a theory over $L(\in L s)$, and let $\varphi, \psi$ be formulas.

(i) (RLDT) $T \cup\{\varphi\} \vdash_{L} \psi$ iff there is $n$ such that $T \vdash_{L} \varphi_{t}^{n} \rightarrow \psi$.

(ii) (RDT) For L with (SIN), $T \cup\{\varphi\} \vdash_{L} \psi$ iff $T \vdash_{L} \varphi_{t} \rightarrow \psi$.

Proof For (i), see Novak [19, Theorem 9, Corollary 1]; (ii) is the enthymematic deduction theorem (see Meyer, Dunn, and Leblanc [18, Lemma 4]).

An easy computation shows the following.

\section{Proposition 2}

(i) $\mathrm{RMAILL}$ proves

(1) $(\psi \rightarrow \chi) \rightarrow((\varphi \rightarrow \psi) \rightarrow(\varphi \rightarrow \chi))$

(prefixing, $P F$ )

(2) $(\varphi \rightarrow \psi) \rightarrow((\varphi \& \chi) \rightarrow(\psi \& \chi))$

(monotonicity, $M T$ )

(3) $(\varphi \rightarrow(\psi \rightarrow \chi)) \rightarrow(\psi \rightarrow(\varphi \rightarrow \chi))$

(permutation, $P M)$

(4) $(\varphi \&(\psi \& \chi)) \rightarrow((\varphi \& \psi) \& \chi)$

(associativity, $A S$ )

(5) $(\varphi \rightarrow \psi) \rightarrow(\neg \psi \rightarrow \neg \varphi)$

(contraposition, $C P$ )

(6) $\varphi \rightarrow \neg \neg \varphi$

(double negation introduction, DNI)

(7) $\neg(\varphi \vee \psi) \leftrightarrow(\neg \varphi \wedge \neg \psi)$

(de MorganI, DMI)

(8) $(\neg \varphi \vee \neg \psi) \rightarrow \neg(\varphi \wedge \psi)$

$(\vee \neg)$

(9) $(\varphi \&(\psi \vee \chi)) \leftrightarrow((\varphi \& \psi) \vee(\varphi \& \chi))$

$(\& \vee$-distributivity, \& $\&-D)$ 
(10) $(\varphi \&(\psi \wedge \chi)) \rightarrow((\varphi \& \psi) \wedge(\varphi \& \chi))$

$(\& \wedge)$

(11) $\left(\varphi_{t} \& \varphi_{t}\right) \rightarrow \varphi_{t}$

$\left(\boldsymbol{t}\right.$-square decreasing, $\left.S D E_{t}\right)$

(12) $\varphi \leftrightarrow(t \rightarrow \varphi)$

(13) $\neg(\varphi \wedge \neg \varphi), \neg \varphi \vee \neg \neg \varphi$

(ii) $\boldsymbol{R} \boldsymbol{U} \boldsymbol{L}$ proves

(1) $(\neg \varphi \vee \neg \psi) \leftrightarrow \neg(\varphi \wedge \psi)$

(2) $(\varphi \&(\psi \wedge \chi)) \leftrightarrow((\varphi \& \psi) \wedge(\varphi \& \chi))$

(de MorganII, DM2)

(3) $(\varphi \rightarrow \psi) \vee(\psi \rightarrow \varphi)$

$(\& \wedge$-distributivity, $\& \wedge-D)$

(4) For each $n,(\varphi \rightarrow \psi)_{t}^{n} \vee(\psi \rightarrow \varphi)_{t}^{n}$

$(P L)$

(5) $(\varphi \wedge(\varphi \rightarrow f)) \rightarrow(\psi \vee(\psi \rightarrow f))$, that is, $(\varphi \wedge \neg \varphi) \rightarrow(\psi \vee \neg \psi)$

(6) $(\varphi \wedge(\psi \vee \chi)) \rightarrow((\varphi \wedge \psi) \vee(\varphi \wedge \chi))$

(distributivity, $D$ )

(iii) RUL having (SIN) proves

(1) $(\varphi \wedge \psi) \rightarrow(\varphi \& \psi)$

(iv) $\boldsymbol{R U M L}$ proves

(1) $(\varphi \& \psi) \rightarrow(\varphi \vee \psi)$

(2) $((\varphi \vee \psi) \rightarrow(\varphi \& \psi)) \vee((\varphi \& \psi) \rightarrow(\varphi \wedge \psi)))$

(3) $((\varphi \& \psi) \leftrightarrow(\varphi \wedge \psi)) \vee((\varphi \& \psi) \leftrightarrow(\varphi \vee \psi))$

(v) RIUL (=FRW) proves

(1) $\varphi \leftrightarrow \neg \neg \varphi$

(double negation, DN)

(vi) $\boldsymbol{F R}$ without (EM) proves

(1) $\varphi \vee \neg \varphi$

(2) $\neg(\varphi \wedge \neg \varphi)$

(excluded middle, EM)

(noncontradiction, $\mathrm{NC}$ )

(vii) $\boldsymbol{R M}(=\boldsymbol{F} \boldsymbol{R} \boldsymbol{M})$ proves

(1) $(\varphi \rightarrow \psi) \rightarrow((\varphi \rightarrow \psi) \leftrightarrow(\neg \varphi \vee \psi))$

(2) $\neg(\varphi \rightarrow \psi) \rightarrow((\varphi \rightarrow \psi) \leftrightarrow(\neg \varphi \wedge \psi))$

(3) $(\varphi \rightarrow \neg \psi) \rightarrow((\varphi \& \psi) \leftrightarrow(\varphi \wedge \psi))$

(4) $\neg(\varphi \rightarrow \neg \psi) \rightarrow((\varphi \& \psi) \leftrightarrow(\varphi \vee \psi))$

(5) $\neg(\varphi \rightarrow \varphi) \rightarrow(\psi \rightarrow \psi)$

(6) $f \rightarrow t$

In $\mathrm{L}(\in \mathrm{Ls})$, the negation $\neg$ may be taken as a primitive connective, and the constant $\mathbf{f}$ can be instead defined as $\neg \mathbf{t}$ (see Proposition 2(i)(12)).

For convenience, $\neg, \wedge, \vee$, and $\rightarrow$ are used ambiguously as propositional connectives and as algebraic operators, but context should clarify their meanings.

2.2 Semantics Suitable algebraic structures for the (fuzzy-)relevance logics are obtained as varieties of residuated lattices in the sense of [11].

Definition 6 A pointed commutative residuated lattice is a structure $(A, t, f, \wedge$, $\vee, *, \rightarrow)$ such that: ${ }^{7}$

(I) $(A, \wedge, \vee)$ is a lattice,

(II) $(A, *, t)$ is a commutative monoid,

(III) $y \leq x \rightarrow z$ iff $x * y \leq z$, for all $x, y, z \in A$ (residuation),

(IV) $f$ is an arbitrary element of $A$.

As $\varphi^{n}$ in Section 2.1, by $x^{n}$, we denote $x * \cdots * x, n$ factors.

Note that the class of pointed commutative residuated lattices characterizes the system $\mathbf{F L} \mathbf{L}_{e}$. Thus, we henceforth call such residuated lattices $\boldsymbol{F} \boldsymbol{L}_{e}$-algebras. 
Definition $7 \quad$ Let $\neg x:=x \rightarrow f$, and let $x_{t}:=x \wedge t$.

(i) (RMAILL-algebra) An RMAILL-algebra is a pointed commutative residuated lattice satisfying the condition

(EM) $t \leq x \vee \neg x$.

(ii) (RUL-algebra) An RUL-algebra is an RMAILL-algebra satisfying the condition

$\left(\mathrm{PL}_{t}\right) t \leq(x \rightarrow y)_{t} \vee(y \rightarrow x)_{t}$.

In an analogy to Definition 7, we can define algebras corresponding to the systems introduced in Definitions 3 and 4. When we define the $\mathrm{FL}_{e}$-algebra, we can use in place of (III) a family of equations as in [11] and [12].

An RMAILL-algebra is said to be linearly ordered if the ordering of its algebra is linear; that is, $x \leq y$ or $y \leq x$ (equivalently, $x \wedge y=x$ or $x \wedge y=y$ ) for each pair $x, y$. Note that, in RMAILL-algebras, the operator $\neg$ can be defined using $\rightarrow$ and $f$, as above. Thus, an RMAILL-algebra $(A, t, f, \neg, \wedge, \vee, *, \rightarrow)$ may be abbreviated to $(A, t, f, \wedge, \vee, *, \rightarrow)$.

As in Section 2.1, for brevity, by L-algebra(s), we henceforth ambiguously express algebras corresponding to all L systems, if we need not distinguish them, but context should determine which algebras are intended.

Definition 8 (Evaluation) Let $\mathcal{A}$ be an L-algebra. An $\mathcal{A}$-evaluation is a function $v: F O R \rightarrow \mathcal{A}$ satisfying: $v(\varphi \rightarrow \psi)=v(\varphi) \rightarrow v(\psi), v(\varphi \wedge \psi)=v(\varphi) \wedge v(\psi)$, $v(\varphi \vee \psi)=v(\varphi) \vee v(\psi), v(\varphi \& \psi)=v(\varphi) * v(\psi), v(\mathbf{t})=t, v(\mathbf{f})=f$ (and hence $v(\neg \varphi)=\neg v(\varphi))$.

Definition 9 ([5, Definitions 16-17]) Let $\mathcal{A}$ be an L-algebra, let $T$ be a theory, let $\varphi$ be a formula, and let $\mathcal{K}$ be a class of L-algebras.

(i) (Tautology) $\varphi$ is a $t$-tautology in $\mathcal{A}$, briefly an $\mathcal{A}$-tautology (or $\mathcal{A}$-valid), if $v(\varphi) \geq t$ for each $\mathcal{A}$-evaluation $v$.

(ii) (Model) An $\mathcal{A}$-evaluation $v$ is an $\mathcal{A}$-model of $T$ if $v(\varphi) \geq t$ for each $\varphi \in T$. By $\operatorname{Mod}(T, \mathcal{A})$, we denote the class of $\mathcal{A}$-models of $T$.

(iii) (Semantic consequence) $\varphi$ is a semantic consequence of $T$ w.r.t. $\mathcal{K}$, denoted by $T \models \mathcal{K} \varphi$, if $\operatorname{Mod}(T, \mathcal{A})=\operatorname{Mod}(T \cup\{\varphi\}, \mathcal{A})$ for each $\mathcal{A} \in \mathcal{K}$.

Definition 10 (L-algebra, [5]) Let $\mathcal{A}, T$, and $\varphi$ be as in Definition $9 . \mathcal{A}$ is an $\boldsymbol{L}$-algebra if, whenever $\varphi$ is L-provable in any $T$ (i.e., $T \vdash_{L} \varphi, \mathrm{L}$ an $\mathrm{L}$ logic), it is a semantic consequence of $T$ w.r.t. $\{\mathcal{A}\}$ (i.e., $T \models_{\{\mathcal{A}\}} \varphi, \mathcal{A}$ a corresponding L-algebra). By $\operatorname{MOD}(L)$, we denote the class of $\mathbf{L}$-algebras; by $M O D^{l}(L)$, the class

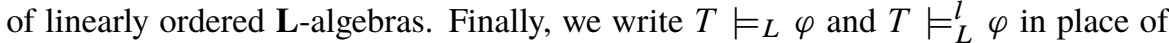
$T \models_{M O D(L)} \varphi$ and $T \models_{M O D^{l}(L)} \varphi$, respectively.

Note that, since each condition for an RMAILL-algebra (more generally, L-algebra) has the form of an equation or can be defined in an equation, it can be ensured that the class of all RMAILL-algebras (L-algebras) is a variety. Then, as in [16], we can show that $\mathrm{L}$ is complete w.r.t. an algebraic semantic given by a variety of L-algebras. We instead show that $\mathrm{L}$ is a weakly implicative fuzzy logic. This also implies the completeness of $\mathrm{L}$.

Let a theory $T$ be linear if, for each pair $\varphi, \psi$ of formulas, $T \vdash \varphi \rightarrow \psi$ or $T \vdash \psi \rightarrow \varphi$. We denote the class of all RUL-algebras as RUL and the class of all $\mathrm{L}$-algebras as $\mathrm{L}$. The system RUL is an algebraizable logic in the sense of Blok and 
Pigozzi (see Czelakowski [6]). Then, since it implies that all axiomatic extensions of RUL are also algebraizable and that their equivalent algebraic semantics are the subvarieties of RUL defined by the translations of the axioms into equations, $\mathrm{L}$ is also algebraizable, and its equivalent algebraic semantic $L$ is the subvariety of RUL.

Cintula [5] defined weakly implicative logic (WIL) as a logic satisfying A1, (mp), transitivity $(\varphi \rightarrow \psi, \psi \rightarrow \chi \vdash \varphi \rightarrow \chi)$, and congruence w.r.t connectives and called a WIL L a fuzzy logic (i.e., a weakly implicative fuzzy logic or WIFL) if it is complete w.r.t. linearly ordered (corresponding) matrices. He also showed that, for a finitary WIL $\mathbf{L}$, the following are equivalent.

(1) $\mathbf{L}$ is a fuzzy logic.

(2) $\mathbf{L}$ has the linear extension property; that is, for each theory $T$, if $T \nvdash \varphi$, then there is a consistent linear theory $T^{\prime} \supseteq T$ such that $T^{\prime} \nvdash \varphi$.

(3) $\mathbf{L}$ has the prelinearity property, that is, for each theory $T$, if $T, \varphi \rightarrow \psi \vdash \chi$ and $T, \psi \rightarrow \varphi \vdash \chi$, then $T \vdash \chi$.

(4) $\mathbf{L}$ has the subdirect decomposition property; that is, each ordered $\mathbf{L}$-matrix is a subdirect product of linearly ordered $\mathbf{L}$-matrices.

We can easily show that the system RMAILL is a WIL. Let a logic extending RMAILL be a relevant multiplicative additive intuitionistic linear logic (briefly, an RMAILL). For an RMAILL $\mathbf{L}$, Proposition 2(ii)(4) is the condition for $\mathbf{L}$ to be a fuzzy logic.

Theorem 1 Let $\boldsymbol{L}$ be an RMAILL. Then $\boldsymbol{L}$ is a fuzzy logic iff, for each $n$, $\vdash_{L}(\varphi \rightarrow \psi)_{t}^{n} \vee(\psi \rightarrow \varphi)_{t}^{n}$.

Proof The left-to-right direction is obvious. For the right-to-left direction, we show that $\mathbf{L}$ has the prelinearity property. Let $T, \varphi \rightarrow \psi \vdash_{\mathbf{L}} \chi$ and $T, \psi \rightarrow \varphi \vdash_{\mathbf{L}} \chi$. By the theorem (RLDT), for some $n, m, T \vdash_{\mathbf{L}}(\varphi \rightarrow \psi)_{\mathbf{t}}^{n} \rightarrow \chi$ and $T \vdash_{\mathbf{L}}(\psi \rightarrow$ $\varphi)_{\mathbf{t}}^{m} \rightarrow \chi$. Let $m \leq n$. Proposition 2(i)(11) ensures that we can obtain $T \vdash_{\mathbf{L}}$ $(\varphi \rightarrow \psi)_{\mathbf{t}}^{n} \rightarrow \chi$ and $T \vdash_{\mathbf{L}}(\psi \rightarrow \varphi)_{\mathbf{t}}^{n} \rightarrow \chi$. Then, by A5 (together with (adj) and $(\mathrm{mp}))$, we get $T \vdash_{\mathbf{L}}\left((\varphi \rightarrow \psi)_{\mathbf{t}}^{n} \vee(\psi \rightarrow \varphi)_{\mathbf{t}}^{n}\right) \rightarrow \chi$. Thus, by Proposition 2(ii)(4) and (mp), $T \vdash_{\mathbf{L}} \chi$, as desired.

Then, from Theorem 1, we establish the following corollaries.

Corollary 1 (Strong completeness) Let $T$ be a theory over $L(\in L s)$, and let $\varphi$ be a formula. Then $T \vdash_{L} \varphi$ iff $T \models_{L}^{l} \varphi$.

Corollary 2 Lis a fuzzy logic (in Cintula's sense).

Now we verify the relevance of $L$.

\section{Theorem 2}

(i) L does not satisfy $\operatorname{SRP}$ (in [1]).

(ii) L satisfies WRP (in [7]).

Proof (i) This directly follows from Proposition 2(ii)(5).

(ii) We prove this contrapositively. Namely, we assume that $\varphi, \psi$ share no propositional variables and either $\nvdash_{L} \neg \varphi$ or $\nvdash_{L} \psi$ and show that $\nvdash_{L} \varphi \rightarrow \psi$. For this, consider an algebra $\mathcal{A}=\left(\left\{1, \frac{1}{2}, 0\right\}, \frac{1}{2}, \frac{1}{2}\right.$, min, max, $\left.*, \rightarrow\right)$, where, letting $\neg x$ (i.e., $\left.x \rightarrow \frac{1}{2}\right)=1-x$,

$$
x * y= \begin{cases}\min (x, y) & \text { if } x \leq \neg y \\ \max (x, y) & \text { otherwise }\end{cases}
$$


and

$$
x \rightarrow y= \begin{cases}\max (\neg x, y) & \text { if } x \leq y, \\ \min (\neg x, y) & \text { otherwise. }\end{cases}
$$

The axioms of $\mathrm{L}$ are $\mathcal{A}$-tautologies for all assignments of values to the variables, and the rules preserve this property. This ensures soundness; that is, if $\vdash_{L} \varphi$, then $\models_{\mathcal{A}} \varphi$. Let $\varphi$ and $\psi$ share no propositional variables, and let either $\nvdash_{L} \neg \varphi$ or $\nvdash_{L} \psi$. If $\vdash_{L} \neg \varphi$ and $\nvdash_{L} \psi$, then assign the values yielding $v(\varphi)=1 / 2$ to all the variables of $\varphi$ and the values yielding $v(\psi)=0$ to all the variables of $\psi$; if $\nvdash_{L} \neg \varphi$ and $\vdash_{L} \psi$, then assign the values yielding $v(\varphi)=1$ to all the variables of $\varphi$ and the values yielding $v(\psi)=1 / 2$ to all the variables of $\psi$; otherwise, that is, if $\nvdash_{L} \neg \varphi$ and $\nvdash_{L} \psi$, assign the values yielding $v(\varphi)=1$ to all the variables of $\varphi$ and the values yielding $v(\psi)=0$ to all the variables of $\psi$. Then, in each case, $v(\varphi \rightarrow \psi)$ is 0 . Thus, $\forall \forall_{\mathcal{A}} \varphi \rightarrow \psi$. Therefore, by soundness, $\nvdash_{L} \varphi \rightarrow \psi$, as required.

Corollary $3 \quad L$ is a relevance logic (in the weak sense of [7]).

Corollary $4 \quad$ Lis both a fuzzy logic and a relevance logic.

2.3 Substructural relevance logics (I) Let RMAILL, RMALL (= LRW plus (EM)), LR, RMAILML, LRM, and RMALML be the systems excluding $\left(\mathrm{PL}_{\mathbf{t}}\right)$ from the systems RUL, RIUL (= FRW), FR, RUML, RM, and RIUML, respectively. We let $L s^{-}$be the set of these systems; that is, we have the following.

\section{Definition $11 L s^{-}=$\{RMAILL, RMALL, LR, RMAILML, LRM, RMALML\}.}

Since $\mathrm{L}^{-}\left(\in \mathrm{Ls}^{-}\right)$is an RMAILL and so a WIL, Theorem 1 shows that $\mathrm{L}(\in \mathrm{Ls})$ is a WIFL (see Corollary 2); moreover, $\mathrm{L}$ is the weakest fuzzy logic extending $\mathrm{L}^{-}$. (Note that the systems LRM and RMALML are not the same as RM and RIUML, respectively, because the former systems exclude (D), i.e., Proposition 2(ii)(6).) More exactly, [5, Theorem 1] says that, for a WIL $\mathbf{L}, T \vdash \varphi$ iff $T \models \varphi$, and so we obtain the following corollary.

Corollary 5 (Strong completeness) For each theory $T$ over $L^{-}\left(\in L s^{-}\right)$and formula $\varphi, T \vdash_{L^{-}} \varphi$ iff $T \models_{L^{-}} \varphi$.

Let us verify the relevance of $\mathrm{L}^{-}\left(\in \mathrm{Ls}^{-}\right)$. Before verifying this, we first note that an easy computation shows the following.

Proposition $3 \quad$ LRM and RMALML each prove $\neg(\varphi \rightarrow \varphi) \rightarrow(\psi \rightarrow \psi)$, that is, Proposition 2(vii)(5).

Corollary 6 For $L^{-} \in\{$ LRM, RMALML $\}, L^{-}$does not satisfy SRP (in [1]).

We can further show the following.

\section{Theorem 3}

(i) For $L^{-} \in\{$ RMAILL, RMALL, RMAILML, LR $\}, L^{-}$satisfies SRP (in [1]).

(ii) For $L^{-} \in\{$ LRM, RMALML $\}, L^{-}$satisfies WRP (in [7]).

Proof (i) First note that $\mathrm{L}^{-}(\in\{$ RMAILL, RMALL, LR $\})$ satisfies the matrices considered in [2] in order to show that $\mathbf{R}^{0}$, the $\mathbf{t}$-free fragment of $\mathbf{R}$, satisfies SRP. Thus, we prove that the system RMAILML satisfies SRP. We assume that $\varphi, \psi$ share no propositional variables and show that $\nvdash_{\text {RMAILML }} \varphi \rightarrow \psi$. For this, con- 
sider the algebra $\mathcal{A}$ in the proof of Theorem 2(ii). The axioms of RMAILML are $\mathcal{A}$-tautologies for all assignments of values to the variables, and the rules preserve this property. This ensures soundness. Let $\varphi$ and $\psi$ share no propositional variables. Assign the values yielding $v(\varphi)=1 / 2$ to all the variables of $\varphi$ and the values yielding $v(\psi)=0$ to all the variables of $\psi$. Then, $v(\varphi \rightarrow \psi)$ is 0 . Thus, $\forall \forall_{\mathcal{A}} \varphi \rightarrow \psi$. Therefore, by soundness, $\nvdash_{\text {RMAILML }} \varphi \rightarrow \psi$, as required.

(ii) This proof is analogous to that of Theorem 2.

Corollary $7 \quad L^{-}$is a relevance logic (in the strong or weak sense of [1], [7]).

Remark 3 As is known, the relevance logics RW, R, RM and fuzzy logics such as UL and BL are all substructural logics extending $\mathbf{F L} \mathbf{L}_{e}$. Since the system RMAILL is the $\mathbf{F L}_{e}$ with (EM), the (fuzzy-)relevance systems introduced in Definitions 5 and 11 are all substructural logics extending $\mathbf{F L} \mathbf{L}_{e}$. Thus, the system RMAILL may instead be expressed as $\mathbf{F L} \mathbf{L}_{e}^{e m}$ (in the substructural logic tradition) and analogously for the other systems. Note that, in the literature of substructural logic, $\mathbf{F} \mathbf{L}_{e c}$ is already introduced as $\mathbf{F L}_{e}$ plus contraction and $\mathbf{R W}$ and $\mathbf{R}$ as $\mathbf{I n D F L}_{e}\left(=\mathbf{F L}_{e}\right.$ plus distributivity and involution) and $\mathbf{I n D F L}_{e c}$, respectively (see [11]). Thus, the introduction of $\mathbf{F L}_{e}^{e m}$ (including $\mathbf{F L} \mathbf{L}_{e}$ ) and its extensions in Definition 11 is a step in the natural evolution of relevance logic (in particular associated with fuzzy logic) in the search for weaker systems.

\section{Propositional Constants and New Relevance Principles}

This section summarizes our work in [22]. More exactly, we briefly recall new strong and weak relevance principles introduced in [22], that is, NSRP and NWRP, because they are unfamiliar to the readers. (As we mentioned in footnote 2, we assumed that formulas such as $(\varphi \wedge \mathbf{t}) \rightarrow(\mathbf{t} \vee \psi)$ satisfy SRP in L $(\in \mathrm{Ls})$ because its antecedent and consequent implicitly share at least one propositional variable. The introduction of NSRP and NWRP will show the reason for this assumption. However, the principles NSRP and NWRP were introduced without such an assumption (see [22]).) Before introducing these principles, we consider intensional features of propositional constants $\mathbf{t}, \mathbf{f}, \mathbf{T}, \mathbf{F}$ and some (defective) weak versions of the new principles.

Many logical systems with propositional constants are equivalent to those without propositional constants because, in the latter systems, propositional constants are definable. For instance, the constants $\mathbf{F}$ and $\mathbf{T}$ can be defined as $\varphi \wedge \neg \varphi$ and $\varphi \vee \neg \varphi$, respectively, in classical logic (CL). However, such equivalences are not valid in the systems $\mathbf{R}$ and $\mathbf{R}^{\mathbf{T}}$ (R plus constants $\mathbf{T}, \mathbf{F}$, and axioms A12, A13 below) because we cannot define the propositional constants $\mathbf{t}, \mathbf{f}$ or $\mathbf{T}, \mathbf{F}$, respectively, using propositional language from which they are excluded.

One interesting fact to mention is that, in the literature of relevance logic (see, e.g., [2], [8]), the constant $\mathbf{t}$ is interpreted as the conjunction of all true sentences, and similarly for the others. More exactly, the propositional constants in $\mathbf{R}$ and $\mathbf{R}^{\mathbf{T}}$ can be interpreted as follows (see Anderson and Belnap [2, Section 27.1.2] and Dunn [8, Section 1.3]): ${ }^{8}$

df3. $\mathbf{t}=$ the conjunction of all true sentences;

df4. $\mathbf{f}=$ the disjunction of all false sentences;

df5. $\mathbf{T}=$ the disjunction of all sentences;

df6. $\mathbf{F}=$ the conjunction of all sentences. 
Here, df 3 and df4 are semantic interpretations. The corresponding syntactic interpretations can be provided as follows: ${ }^{9}$

$\mathrm{df}^{\prime} . \mathbf{t}=$ the conjunction of all theorems;

df $4^{\prime} . \mathbf{f}=$ the negation of $\mathbf{t}$.

These are not object-definitions, but metadefinitions, which cannot be provided by means of the object-language for the $\mathbf{t}$-free fragment of $\mathbf{R}$ (calling it here $\boldsymbol{R}^{0}$ ). That is, the propositional constants $\mathbf{t}, \mathbf{f}, \mathbf{T}, \mathbf{F}$ are not object-definable in $\mathbf{R}^{0} ; \mathbf{T}, \mathbf{F}$ are not in $\mathbf{R}$. Thus, we cannot eliminate those constants in $\mathbf{R}$ or $\mathbf{R}^{\mathbf{T}}$. (We use the word object-definition for a clear distinction from metadefinition.) Although df3 to df6, $\mathrm{df}^{\prime}$, and df4' are not object-definitions of propositional constants, they cannot be defined as we please because the definitions are based on (syntactic and semantical) roles of the constants in the proof of soundness and completeness for a relevance logic. In fact, the above interpretations are given according to such roles of the constants in $\mathbf{R}$ and $\mathbf{R}^{\mathbf{T}}$. (Note that, as mentioned in [22], the propositional constants $\mathbf{t}, \mathbf{T}, \mathbf{f}, \mathbf{F}$ correspond to the least true element $t$, the greatest (true) element $T$, and their negations, i.e., the greatest false element $f$, and the least (false) element $\perp$, respectively, in algebraic semantics; see [2], [7], [8].)

The relevance principle is a metacriterion or metaprinciple, by virtue of which we can examine whether a system is a relevance logic. Similarly, metadefinitions or interpretations can be used to assess variable sharing between the antecedent and consequent of an implication (as a theorem), as far as they are given as above (i.e., according to syntactic and semantical roles of the constants). Let metadefinitions of propositional constants be given like this, that is, based on the above roles of constants; let the antecedent and consequent $\varphi, \psi$ of a statement $\varphi \rightarrow \psi$ implicitly share a propositional variable by virtue of the metadefinitions. For example, in $\mathbf{R}$, the antecedent $p \wedge \mathbf{t}$ and the consequent $\mathbf{t} \vee q$ of the theorem $(p \wedge \mathbf{t}) \rightarrow(\mathbf{t} \vee q)$ implicitly share (the conjunction of ) "all true sentences (or theorems)" by virtue of df3 (or df3').

Before introducing new relevance principles, we introduce their weak versions and related facts in order to help the readers better understand them.

\section{Definition 12 ([22, Definition 6])}

(i) (The implicit strong relevance principle, ISRP) $\varphi \rightarrow \psi$ is a theorem only if $\varphi$ and $\psi$ implicitly share a propositional variable where the word implicitly means that we can identify a sharing variable by means of metadefinitions or interpretations such as df 3 to df6, df3', and df4'.

(ii) (The implicit weak relevance principle, IWRP) $\varphi \rightarrow \psi$ is a theorem only if (a) $\varphi$ and $\psi$ implicitly share a propositional variable or (b) both $\neg \varphi$ and $\psi$ are theorems.

Let $\varphi \rightarrow \psi$ satisfy the relevance principle ISRP (resp., IWRP) in a logic $\mathbf{L}$ if it is a theorem of $\mathbf{L}$ and its antecedent $\varphi$ and consequent $\psi$ implicitly share a propositional variable (or both the negation of its antecedent and its consequent are theorems). Then we can prove the following.

\section{Proposition 4 (see [22, Proposition 4])}

(i) $(\varphi \wedge \boldsymbol{t}) \rightarrow(\boldsymbol{t} \vee \psi)$ satisfies ISRP in $\boldsymbol{R}$.

(ii) $((\varphi \rightarrow \boldsymbol{F}) \& \varphi) \rightarrow \psi$ satisfies ISRP in $\boldsymbol{R}^{\boldsymbol{T}}$ and $\boldsymbol{U} \boldsymbol{L}$.

(iii) $((\varphi \rightarrow \boldsymbol{F}) \wedge \varphi) \rightarrow \psi$ satisfies ISRP in $\boldsymbol{R}^{T}$. 
(iv) $((\varphi \rightarrow \varphi) \rightarrow \boldsymbol{f}) \rightarrow(\psi \rightarrow \psi)$ satisfies ISRP in IUML.

(v) $(\varphi \wedge \neg \varphi) \rightarrow(\psi \vee \neg \psi)$ satisfies IWRP in $\boldsymbol{R M}$.

(vi) $\neg(\varphi \rightarrow \varphi) \rightarrow(\psi \rightarrow \psi)$ satisfies IWRP in $\boldsymbol{R} \boldsymbol{M}$ and $\boldsymbol{R} \boldsymbol{M}^{\boldsymbol{T}}$.

Proof We can easily prove (i) to (v) using df3 (or df3'), df4 (or df4'), and df6. Then, (vi) follows from Proposition 2(vii)(5), Proposition 5(iv)(1) and the second condition of IWRP.

The principles ISRP and IWRP, however, do not prevent us from giving metadefinitions of propositional constants to the systems having their object-definitions. (Note that the constants $\mathbf{T}$ and $\mathbf{F}$ can still be interpreted as df5 and df6, respectively, in CL.) For instance, if CL has not only the object-definitions (df7) $\mathbf{T}:=\varphi \vee \neg \varphi$, (df8) $\mathbf{F}:=\varphi \wedge \neg \varphi$, but also the metadefinitions df5, df6, then the statement $(p \wedge \neg p) \rightarrow q$ satisfies ISRP and IWRP in CL since the sentences $\mathbf{T}, \mathbf{F} \rightarrow \mathbf{F}$, and $\mathbf{T} \leftrightarrow(\mathbf{F} \rightarrow \mathbf{F}) \leftrightarrow((p \wedge \neg p) \rightarrow q)$ are all theorems of CL. (We generally regard the statement $(p \wedge \neg p) \rightarrow q$ as an example of paradoxes of material implication, and many irrelevant logics such as CL, intuitionistic logic, etc., can allow (2), from which an irrelevance between the antecedent and consequent of an implication arises.)

Note that a propositional constant in a logic $\mathbf{L}$ is said to be object-definable in that it can be defined by means of the object-language for $\mathbf{L}$ and metadefinable in that it can be defined by virtue of metadefinitions such as df3 to df6, df3', and df4'. Let a propositional constant be strongly metadefinable in a logic $\mathbf{L}$ if it is metadefinable but not object-definable in $\mathbf{L}$, for example, the constants $\mathbf{t}$ and $\mathbf{f}$ in $\mathbf{R}$ and $\mathbf{T}$ and $\mathbf{F}$ in $\mathbf{R}^{\mathbf{T}}$; let the antecedent and consequent of an implication strong implicitly share a propositional variable if we can establish variable sharing between them by virtue of strong metadefinitions.

The new strong and weak relevance principles introduced in [22] are defined as follows. ${ }^{10}$

\section{Definition 13 ([22, Definition 8])}

(i) (The new strong relevance principle, NSRP) $\varphi \rightarrow \psi$ is a theorem only if $\varphi$ and $\psi$ either explicitly or strong implicitly share a propositional variable.

(ii) (The new weak relevance principle, NWRP) $\varphi \rightarrow \psi$ is a theorem only if (i) $\varphi$ and $\psi$ either explicitly or strong implicitly share a propositional variable, or (ii) both $\neg \varphi$ and $\psi$ are theorems.

The principles NSRP and NWRP prevent logics with both object-definable and metadefinable propositional constants from satisfying the relevance principles. For instance, CL satisfies neither NSRP nor NWRP since, in CL, the constants $\mathbf{T}$ and $\mathbf{F}$ can be defined not merely by df7 and df8, but by df5 and df6.

\section{Fuzzy-Relevance Logics (II)}

4.1 Fuzzy-relevance logics with constants T, F In this section, we introduce several substructural fuzzy-relevance systems satisfying new relevance principles, that is, NSRP and NWRP. First, we provide axiomatizations of the $\mathrm{L}$ with constants T, F.

\section{Definition 14}

(i) UL is RUL minus (EM) plus constants $\mathbf{F}$, T, and A12. $\mathbf{F} \rightarrow \varphi$

A13. $\varphi \rightarrow$ T 
(ii) IUL ( $=$ FRW $\left.^{\mathbf{T}}\right)$ is UL plus (DNE).

(iii) $\mathbf{F R}^{\mathbf{T}}$ is IUL plus (SIN).

(iv) UML is UL plus (ID).

(v) $\mathbf{R M}^{\mathbf{T}}$ is $\mathbf{U M L}$ plus (DNE).

(vi) IUML is $\mathbf{R M}^{\mathbf{T}}$ plus (FP).

\section{Definition $15 \quad \mathrm{Ls}^{\mathbf{T}}=\left\{\mathbf{U L}, \mathbf{I U L}\left(=\mathbf{F R W}^{\mathbf{T}}\right), \mathbf{F R}^{\mathbf{T}}, \mathbf{U M L} \mathbf{R M}^{\mathbf{T}}, \mathbf{I U M L}\right\}$.}

An easy computation shows the following.

\section{Proposition 5}

(i) $L(\in L s)$ proves

(1) $(\varphi \wedge \boldsymbol{t}) \rightarrow(\boldsymbol{t} \vee \psi)$.

(ii) $L^{T}\left(\in L s^{T}\right)$ proves

(1) $(\varphi \wedge \neg \varphi) \rightarrow(\psi \vee \neg \psi)$;

(2) $((\varphi \rightarrow \boldsymbol{F}) \& \varphi) \rightarrow \psi$.

(iii) $\boldsymbol{F R} \boldsymbol{R}^{\boldsymbol{T}}, \boldsymbol{U M L}, \boldsymbol{R} \boldsymbol{M}^{\boldsymbol{T}}$, and IUML each prove

(1) $((\varphi \rightarrow \boldsymbol{F}) \wedge \varphi) \rightarrow \psi$.

(iv) $\boldsymbol{R} \boldsymbol{M}^{\boldsymbol{T}}$ and IUML each prove

(1) $\neg(\varphi \rightarrow \varphi) \rightarrow(\psi \rightarrow \psi)$.

First, note that, using the standard technique, we can provide algebraic completeness results for $\mathrm{L}^{\mathbf{T}}\left(\in \mathrm{Ls}^{\mathbf{T}}\right)$. For this, it suffices to note that $\mathrm{L}^{\mathbf{T}}$-algebras are obtained as varieties of pointed bounded commutative residuated lattices; that is, for $\mathrm{L}^{\mathrm{T}}$-algebras, it suffices to replace the condition (I) in Definition 6 with

$\left(\mathrm{I}^{\prime}\right)(A, \top, \perp, \wedge, \vee)$ is a bounded lattice with top element $\top$ and bottom element $\perp$.

Since the condition $\left(\mathrm{I}^{\prime}\right)$ can be defined in equations, it is clear that the class of $\mathrm{L}^{\mathbf{T}}$-algebras forms a variety. Then, as in [16], we can show that $\mathrm{L}^{\mathbf{T}}$ is complete w.r.t. an algebraic semantic given by a variety of $\mathrm{L}^{\mathbf{T}}$-algebras. Moreover, as in Section 2.2, we can prove the following.

Theorem $4 \quad$ Let $\boldsymbol{L}$ be a multiplicative additive intuitionistic linear logic. Then $\boldsymbol{L}$ is a fuzzy logic iff, for each $n, \vdash_{L}(\varphi \rightarrow \psi)_{t}^{n} \vee(\psi \rightarrow \varphi)_{t}^{n}$.

Proof See Theorem 1.

Corollary 8 (Strong completeness) Let $T$ be a theory over $L^{T}\left(\in L s^{T}\right)$, and let $\varphi$ be a formula. Then $T \vdash_{L^{T}} \varphi$ iff $T \models_{L^{T}}^{l} \varphi$.

Corollary $9 \quad L^{T}$ is a fuzzy logic (in Cintula's sense).

For the relevance of $\mathrm{L}^{\mathbf{T}}$, note that the constants $\mathbf{T}, \mathbf{F}, \mathbf{t}, \mathbf{f}$ are not object-definable in $\mathrm{L}^{\mathbf{T}}\left(\in \mathrm{Ls}^{\mathbf{T}}\right)$ without the constants. Note also that, in $\mathrm{L}^{\mathbf{T}}$, the constant $\mathbf{t}$ can instead be interpreted as df $3^{\prime}$ (or df3) and the constant $\mathbf{f}$ as its negation (see df 2 and Proposition 2(i)(12)); and the constants $\mathbf{T}$ and $\mathbf{F}$ as df5 and df6, respectively (see A12 and A13 in Definition 14). Then, we can show the following.

\section{Proposition 6}

(i) $(\varphi \wedge \boldsymbol{t}) \rightarrow(\boldsymbol{t} \vee \psi)$ satisfies NSRP in $L(\in L s)$ and $L^{\boldsymbol{T}}\left(\in L s^{\boldsymbol{T}}\right)$.

(ii) $(\varphi \wedge(\varphi \rightarrow f)) \rightarrow(\psi \vee(\psi \rightarrow f))$ satisfies NSRP in $L^{\boldsymbol{T}}\left(\in L s^{T}\right)$.

(iii) $((\varphi \rightarrow \boldsymbol{F}) \& \varphi) \rightarrow \psi$ satisfy NSRP in $L^{\boldsymbol{T}}\left(\in L s^{\boldsymbol{T}}\right)$. 
(iv) $((\varphi \rightarrow \boldsymbol{F}) \wedge \varphi) \rightarrow \psi$ satisfy NSRP in $L^{\boldsymbol{T}} \in\left\{\boldsymbol{F} \boldsymbol{R}^{\boldsymbol{T}}, \boldsymbol{U M} \boldsymbol{M}, \boldsymbol{R} \boldsymbol{M}^{\boldsymbol{T}}, \boldsymbol{I U M L}\right\}$.

(v) $\neg(\varphi \rightarrow \varphi) \rightarrow(\psi \rightarrow \psi)$ satisfies NSRP in IUML.

(vi) $\neg(\varphi \rightarrow \varphi) \rightarrow(\psi \rightarrow \psi)$ satisfies NWRP in $\boldsymbol{R M}^{\boldsymbol{T}}$.

Proof See [22, Proposition 5].

Proposition 6 shows that theorems strong implicitly sharing variable(s) can satisfy NSRP. Thus, we verify the relevance of $\mathrm{L}^{\mathbf{T}}\left(\in \mathrm{Ls}^{\mathbf{T}}\right)$ as follows.

\section{Theorem 5}

(i) For $L^{T} \in L s^{T} \backslash\left\{\boldsymbol{R M} \boldsymbol{M}^{T}\right\}, L^{T}$ satisfies NSRP.

(ii) $\boldsymbol{R} \boldsymbol{M}^{\boldsymbol{T}}$ satisfies $N W R P$.

Proof (i) We can prove this by using the matrices considered in Theorem 2 w.r.t UML and IUML. Let $\mathrm{L} \in\left\{\mathbf{U L}, \mathbf{I U L}, \mathbf{F R}^{\mathbf{T}}\right\}$. Contrapositively, assume that $\varphi$ and $\psi$ share neither propositional variables nor propositional constants and show that $\nvdash_{L} \varphi \rightarrow \psi$. For this, consider an algebra $\mathcal{A}=\left(\left\{1, \frac{5}{6}, \frac{2}{3}, \frac{1}{2}, \frac{1}{3}, \frac{1}{6}, 0\right\}, 1,0\right.$, $\frac{1}{3}, \frac{2}{3}$, min, $\left.\max , *, \rightarrow\right)$, where: Letting $\neg x\left(\right.$ i.e., $\left.x \rightarrow \frac{2}{3}\right)=1-x$,

\begin{tabular}{c|ccccccc}
$\rightarrow$ & $1^{+}$ & $\frac{5}{6}^{+}$ & $\frac{2}{3}^{+}$ & $\frac{1}{2}^{+}$ & $\frac{1}{3}^{+}$ & $\frac{1}{6}$ & 0 \\
\hline $1^{+}$ & 1 & 0 & 0 & 0 & 0 & 0 & 0 \\
$\frac{5}{6}^{+}$ & 1 & $\frac{5}{6}$ & $\frac{1}{6}$ & $\frac{1}{6}$ & $\frac{1}{6}$ & $\frac{1}{6}$ & 0 \\
$\frac{2}{3}^{+}$ & 1 & $\frac{5}{6}$ & $\frac{1}{3}$ & $\frac{1}{6}$ & $\frac{1}{6}$ & $\frac{1}{6}$ & 0 \\
$\frac{1}{2}^{+}$ & 1 & $\frac{5}{6}$ & $\frac{1}{2}$ & $\frac{1}{2}$ & $\frac{1}{6}$ & $\frac{1}{6}$ & 0 \\
$\frac{1}{3}^{+}$ & 1 & $\frac{5}{6}$ & $\frac{2}{3}$ & $\frac{1}{2}$ & $\frac{1}{3}$ & $\frac{1}{6}$ & 0 \\
$\frac{1}{6}^{6}$ & 1 & $\frac{5}{6}$ & $\frac{5}{6}$ & $\frac{5}{6}$ & $\frac{5}{6}$ & $\frac{5}{6}$ & 0 \\
0 & 1 & 1 & 1 & 1 & 1 & 1 & 1 \\
$*$ & 1 & $\frac{5}{6}^{+}$ & $\frac{2}{3}^{+}$ & $\frac{1}{2}^{+}$ & $\frac{1}{3}$ & $\frac{1}{6}$ & 0 \\
\hline $1^{+}$ & 1 & 1 & 1 & 1 & 1 & 1 & 0 \\
$\frac{5}{6}^{+}$ & 1 & $\frac{5}{6}$ & $\frac{5}{6}$ & $\frac{5}{6}$ & $\frac{5}{6}$ & $\frac{1}{6}$ & 0 \\
$\frac{2}{3}^{+}$ & 1 & $\frac{5}{6}$ & $\frac{5}{6}$ & $\frac{5}{6}$ & $\frac{2}{3}$ & $\frac{1}{6}$ & 0 \\
$\frac{1}{2}^{+}$ & 1 & $\frac{5}{6}$ & $\frac{5}{6}$ & $\frac{1}{2}$ & $\frac{1}{2}$ & $\frac{1}{6}$ & 0 \\
$\frac{1}{3}^{+}$ & 1 & $\frac{5}{6}$ & $\frac{2}{3}$ & $\frac{1}{2}$ & $\frac{1}{3}$ & $\frac{1}{6}$ & 0 \\
$\frac{1}{6}^{+}$ & 1 & $\frac{1}{6}$ & $\frac{1}{6}$ & $\frac{1}{6}$ & $\frac{1}{6}$ & $\frac{1}{6}$ & 0 \\
0 & 0 & 0 & 0 & 0 & 0 & 0 & 0
\end{tabular}

In each matrix, for $\rightarrow$ and $*$, the superscript + indicates designated values. The axioms of $\mathrm{L}$ are $\mathcal{A}$-tautologies for all assignments of values to the variables, and the rules preserve this property. This ensures soundness; that is, if $\vdash_{L} \varphi$, then $\models_{\mathcal{A}} \varphi$. Let $\varphi$ and $\psi$ share neither propositional variables nor propositional constants. If $\vdash_{L} \psi$, assign the values yielding $v(\varphi)>1 / 3$ to all the variables of $\varphi$ and the values yielding $v(\psi)=1 / 3$ to all the variables of $\psi$. Otherwise, that is, if $\nvdash_{L} \psi$, assign the values yielding $v(\varphi)=1 / 3$ to all the variables of $\varphi$ and the values yielding $v(\psi)<1 / 3$ to all the variables of $\psi$. Then, in each case, $v(\varphi \rightarrow \psi)$ is $1 / 6$ or 0 . Thus, $\forall \forall_{\mathcal{A}} \varphi \rightarrow \psi$. Therefore, by soundness, $\nvdash_{L} \varphi \rightarrow \psi$, as required.

(ii) This proof is analogous to that of Theorem 2. 


\section{Corollary 10}

(i) For $L^{T} \in L s^{T} \backslash\left\{R M^{T}\right\}, L^{T}$ is a relevance logic (in the new strong sense of [22]).

(ii) $\boldsymbol{R} \boldsymbol{M}^{\boldsymbol{T}}$ is a relevance logic (in the new weak sense of [22]).

Then, from Corollaries 9 and 10, the next corollary directly follows.

Corollary $11 \quad L^{T}$ is both a fuzzy logic and a relevance logic.

4.2 Strong relevant companions The system IUML is $\mathbf{R M}^{\mathbf{T}}$ plus (FP). Here, we note that, while IUML satisfies NSRP, $\mathbf{R M}^{\mathbf{T}}$ does not. This fact gives an insight into a method to obtain relevant companions of weakening-free uninorm (based) logics. We introduce here a way to obtain strong relevant companions of weakening-free logics extending $\mathbf{U L}$. Let $\mathrm{L}$ be the system $\mathbf{U L}$.

Definition 16 We introduce several extensions of $\mathrm{L}$ as follows.

- Involutive L IL is L plus (DNE) $\neg \neg \varphi \rightarrow \varphi$.

- Square increasing $\mathrm{L} \mathrm{L}_{c}$ is $\mathrm{L}$ plus $(\mathrm{SIN}) \varphi \rightarrow(\varphi \& \varphi)$.

- Involutive $\mathrm{L}_{c} \mathrm{IL}_{c}$ is $\mathrm{L}_{c}$ plus (DNE).

- Square decreasing $\mathrm{L} \mathrm{L}_{p}$ is L plus (SDE) $(\varphi \& \varphi) \rightarrow \varphi$.

- Involutive $\mathrm{L}_{p} \mathrm{IL}_{p}$ is $\mathrm{L}_{p}$ plus (DNE).

- Idempotent $\mathrm{L} \mathrm{L} \mathrm{L}_{c p}$ is $\mathrm{L}_{c}$ plus (SDE).

- Involutive $\mathrm{L}_{c p} \mathrm{IL}_{c p}$ is $\mathrm{L}_{c p}$ plus (DNE).

Tables 3 and 4 summarize some axiom schemes and the extensions of UL introduced in Definition 16.

\section{Definition 17}

(i) $\mathrm{ELs}=\left\{\mathrm{L}, \mathrm{IL}, \mathrm{L}_{c}, \mathrm{IL}_{c}, \mathrm{~L}_{p}, \mathrm{IL}_{p}, \mathrm{~L}_{c p}, \mathrm{IL}_{c p}\right\}$.

(ii) For EL $(\in \mathrm{ELs}), \mathrm{EL}^{f}$ is EL plus (FP).

(iii) $\mathrm{ELs}^{f}=\left\{\mathrm{L}^{f}, \mathrm{IL}^{f}, \mathrm{~L}_{c}^{f}, \mathrm{IL}_{c}^{f}, \mathrm{~L}_{p}^{f}, \mathrm{IL}_{p}^{f}, \mathrm{~L}_{c p}^{f}, \mathrm{IL}_{c p}^{f}\right\}$.

Table 3 Some axiom schemes in fuzzy-relevance logics.

\begin{tabular}{|l|l|}
\hline Axiom schema & Name \\
\hline$\neg \neg A \rightarrow A$ & Double negation elimination (DNE) \\
\hline$A \rightarrow A \& A$ & Square increasing (SIN) \\
\hline$A \& A \rightarrow A$ & Square decreasing (SDE) \\
\hline$A \& A \leftrightarrow A$ & Idempotence (ID) \\
\hline
\end{tabular}

Table 4 Some extensions of L (= UL) obtained by adding the corresponding additional axiom schemes.

\begin{tabular}{|l|l|}
\hline Logic & Additional axiom schemes \\
\hline $\mathrm{IL}$ & $(\mathrm{DNE})$ \\
\hline $\mathrm{L}_{c}$ & $(\mathrm{SIN})$ \\
\hline $\mathrm{IL}_{c}$ & $(\mathrm{DNE})$ and (SIN) \\
\hline $\mathrm{L}_{p}$ & $(\mathrm{SDE})$ \\
\hline $\mathrm{IL}_{p}$ & $(\mathrm{DNE})$ and $(\mathrm{SDE})$ \\
\hline $\mathrm{L}_{c p}$ & $(\mathrm{ID})(=(\mathrm{SIN})+(\mathrm{SDE}))$ \\
\hline $\mathrm{IL}_{c p}$ & $(\mathrm{DNE})$ and (ID) \\
\hline
\end{tabular}


Proposition $7 \quad((\varphi \rightarrow \varphi) \rightarrow f) \rightarrow(\psi \rightarrow \psi)$ satisfies NSRP in each of $L_{p}^{f}, I L_{p}^{f}$, $L_{c p}^{f}$, and $I L_{c p}^{f}$.

Proof The proof is immediate since (FP) $\mathbf{t} \leftrightarrow \mathbf{f}$ is an axiom in each system, and so the antecedent and consequent of $((\varphi \rightarrow \varphi) \rightarrow \mathbf{f}) \rightarrow(\psi \rightarrow \psi)$ implicitly share the statement $\psi \rightarrow \psi$.

Theorem 6 For $E L^{f} \in E L s^{f}, E L^{f}$ satisfies NSRP.

Furthermore, as in Section 2, we can prove the completeness of $\mathrm{EL}^{f}$.

Theorem 7 (Strong completeness) Let $T$ be a theory over $E L^{f}\left(\in E L s^{f}\right)$, and let $\varphi$ be a formula. Then $T \vdash_{E L^{f}} \varphi$ iff $T \models_{E L^{f}}^{l} \varphi$.

From Theorems 6 and 7, we obtain the following corollary.

Corollary $12 E L^{f}$ is both a fuzzy logic (in Cintula's sense) and a relevance logic (in the new strong sense of [22]).

4.3 Substructural relevance logics (II) Let MAILL, MALL $\left(=\mathbf{L R W}^{\mathbf{T}}\right), \mathbf{L R}^{\mathbf{T}}$, MAILML, LRM ${ }^{\mathbf{T}}$, and MALML be the systems eliminating $\left(\mathrm{PL}_{\mathbf{t}}\right)$ from the systems UL, IUL $\left(=\mathbf{F R} \mathbf{W}^{\mathbf{T}}\right), \mathbf{F R}^{\mathbf{T}}, \mathbf{U M L}, \mathbf{R M}^{\mathbf{T}}$, and IUML, respectively. We let $\mathrm{Ls}^{\mathbf{T}-}$ be the set of these systems; that is, we have the following.

Definition $18 \mathrm{Ls}^{\mathrm{T}-}=\left\{\right.$ MAILL, MALL, $\mathbf{L R}^{\mathrm{T}}$, MAILML, LRM ${ }^{\mathrm{T}}$, MALML $\}$.

Since $\mathrm{L}^{\mathbf{T}-}\left(\in \mathrm{Ls}^{\mathbf{T}-}\right)$ is a WIL, Theorem 4 shows that $\mathrm{L}^{\mathbf{T}}\left(\in \mathrm{Ls}^{\mathbf{T}}\right)$ is a WIFL (see Corollary 9); moreover, $\mathrm{L}^{\mathbf{T}}$ is the weakest fuzzy logic extending $\mathrm{L}^{\mathbf{T}}-$. More exactly, [5, Theorem 1] says that, for a WIL $\mathbf{L}, \mathrm{T} \vdash \varphi$ iff $\mathrm{T} \models \varphi$, and so we obtain the following corollary.

Corollary 13 (Strong completeness) For each theory $T$ over $L^{T}\left(\in L s^{T-}\right)$ and formula $\varphi, T \vdash_{L^{T-}} \varphi$ iff $T \models_{L^{T-}} \varphi$.

Let us verify the relevance of $\mathrm{L}^{\mathbf{T}-}\left(\in \mathrm{Ls}^{\mathbf{T}-}\right)$. However, we first note that an easy computation shows the following.

Proposition $8 \quad$ LRM $^{\boldsymbol{T}}$ and MALML each prove $\neg(\varphi \rightarrow \varphi) \rightarrow(\psi \rightarrow \psi)$, that is, Proposition 2(vii)(5).

Corollary $14 \quad$ LRM $^{\boldsymbol{T}}$ does not satisfy NSRP (in [22]).

Furthermore, as in Section 4.1, we can show the following.

\section{Theorem 8}

(i) For $L^{T-} \in\left\{\right.$ MAILL, MALL, LR $\boldsymbol{R}^{T}$, MAILML, MALML $\}, L^{-}$satisfies NSRP (in [22]).

(ii) $\boldsymbol{L R M}^{\boldsymbol{T}}$ satisfies NWRP (in [22]).

\section{Proof}

(i) The proof is immediate since $\mathrm{L}^{\mathbf{T}-}$ also satisfies the matrices considered in [2] in order to show that the system $\mathbf{R}^{0}$ satisfies SRP (w.r.t. MAILL, MALL, and $\mathbf{L R}^{\mathbf{T}}$ ) or the matrices considered in Theorem 2 (w.r.t. MAILML and MALML).

(ii) This proof is analogous to that of Theorem 2. 


\section{Corollary $15 \quad L^{T-}$ is a relevance logic (in the new strong or weak sense of [22]).}

Remark 4 Since the system MAILL is the $\mathbf{F L}_{e \perp}$, the (fuzzy-)relevance systems introduced in Definitions 15 and 18 are all substructural logics extending $\mathbf{F L}_{e \perp}$. Thus, the introduction of $\mathbf{F L}_{e \perp}$ and its extensions in Definition 18 is also a step in the natural evolution of relevance logic (in particular associated with fuzzy logic) in the search for weaker systems.

\section{Concluding Remarks}

We introduced several fuzzy-relevance logics with and without constants $\mathbf{T}, \mathbf{F}$ and provided completeness results for them by showing that such logics are WIFLs. We furthermore proved that they satisfy old and new relevance principles. In addition, we considered relevance logics obtained from the fuzzy-relevance logics by omitting prelinearity. All of the systems investigated here are extensions of the substructural $\operatorname{logic} \mathbf{F L} \mathbf{L}_{e}$, and so they are all substructural logics. They also have the associative intensional conjunction (so-called fusion) \&. Therefore, such systems all can be called associative (fuzzy-) relevance logics.

The fuzzy-relevance logics without constants $\mathbf{T}$ and $\mathbf{F}$ are not characterized by models based on uninorms. Note that the uninorm-based systems introduced in [16] have constants $\mathbf{T}$ and $\mathbf{F}$, and the systems with $\mathbf{T}$ and $\mathbf{F}$ investigated here are not relevant in the old senses. This implies that, as far as uninorm (based) systems have $\mathbf{T}$ and $\mathbf{F}$, they cannot be relevant in the old senses and so are not fuzzy-relevance logics in the old senses.

\section{Notes}

1. For the introduction of propositional constants $\mathbf{t}, \mathbf{f}, \mathbf{T}$, and $\mathbf{F}$, see Sections 2 and 4 . In particular, in order to interpret these constants in relevance logic, see Definitions 3-6 in Section 3 .

2. While Sugihara matrices (as a semantic for RM) need not have a fixed point, such matrices on $[0,1]$ (as a semantic for IUML) have such a point, for example, $1 / 2$ in the standard involutive negation $1-x$ so that the logic IUML requires the corresponding axiom (FP) (see [7], [16]). Note that Sugihara matrices with an odd number of elements introduced in [7] have a fixed point corresponding to (FP).

3. Here, we regard $\mathbf{R}^{\mathbf{t}}$ (the $\mathbf{R}$ with the constant $\mathbf{t}$ ) as $\mathbf{R}$. Often in the literature of relevance logic, $\mathbf{R}$ is used for the $\mathbf{t}$-free fragment of $\mathbf{R}^{\mathbf{t}}$. One reason for that is that $\mathbf{R}^{\mathbf{t}}$ proves formulas such as $(\gamma)(\varphi \wedge \mathbf{t}) \rightarrow(\mathbf{t} \vee \psi)$ and so seems not to satisfy the old relevance principles (see [11]). However, we have to mention that, in the literature of relevance logic (e.g., Anderson and Belnap [2]), the constant $\mathbf{t}$ is interpreted as the conjunction of all true sentences. Thus, $(\gamma)$ does implicitly satisfy SRP, and so the relevance principles in a sense do not fail in $\mathbf{R}^{\mathbf{t}}$. Hence, here we assume that such formulas satisfy SRP. We will, in Section 3, introduce NSRP and NWRP as principles allowing implicit variable sharing.

4. This is not a necessity, however, as some logics, for example, psBL (pseudo-BL) and psMTL (pseudo-MTL), are not (see Hájek [13], [14]). 
5. The systems RMAILL and MAILL are the $\mathbf{F L}_{e}$ with (EM) and $\mathbf{F L}_{e \perp}$, respectively (see [11]). While RMAILL does not prove formulas such as $(\delta)((\varphi \rightarrow \mathbf{F}) \& \varphi) \rightarrow \psi$, MAILL does. Thus, the system MAILL is neither strongly nor weakly relevant in the old senses. Here, we introduce RMAILL as a relevant companion of MAILL. Note that the system $\mathbf{F L}_{e}$ does not prove $(\delta)$ and so is strongly relevant in the old sense. Then, we may establish $\mathbf{F L}_{e}$ as the basic relevance logic. However, if this is so, RUL must require not merely $\left(\mathrm{PL}_{\mathbf{t}}\right)$, but the additional axiom (EM), which is irrelevant to fuzziness. Here, we want to use RUL as the weakest fuzzy logic obtained from some weakly implicative logic by adding $\left(\mathrm{PL}_{\mathbf{t}}\right)$ (but no more).

6. Roughly speaking, in IUML, $\mathbf{t}$ and $\mathbf{f}$ both correspond to a fixed point, for example, the element $1 / 2$ in the real unit interval $[0,1]$, because the involutive negation $\neg x$ may be defined as $1-x$ (see [16, Proposition 19]). Since $\mathbf{t}=\neg \mathbf{f}$ in involutive uninorm logics, the sentence $\mathbf{t} \leftrightarrow \mathbf{f} \leftrightarrow \neg \mathbf{f}$ is a theorem in RIUML.

7. A lattice does not have to have top and bottom elements $T$ and $\perp$, and so $t$ and $f$ need not be the same as $T$ and $\perp$, respectively, in pointed commutative residuated lattices. Note that lattices having $T$ and $\perp$ are called bounded lattices (see ( $\mathrm{I}^{\prime}$ ) in Section 4).

8. Here, "true sentences" correspond to t-tautologies in $\mathcal{A}$, that is, $\mathcal{A}$-tautologies, as in Section 2.2. Note that, if propositional quantification is possible, each constant can be defined as follows: $\mathbf{t}:=(\forall p)(p \rightarrow p), \mathbf{f}:=(\exists p) \neg(p \rightarrow p), \mathbf{T}:=(\exists p) p$, and $\mathbf{F}:=(\forall p) p$ (see Anderson, Belnap, and Dunn [3] and Beal and Restall [4]).

9. The interpretation $\mathrm{df}^{\prime}{ }^{\prime}$ is given, for example, in [8, p. 131]. The constant $\mathbf{t}$ is generally interpreted as in endnote 8 (see Meyer [17, p. 173]). We use df3' here because it corresponds more exactly to $\mathrm{df} 3$ when we consider the soundness and completeness of $\mathbf{R}$.

10. Here, the phrase " $\varphi$ and $\psi$ explicitly share a propositional variable" is the same as " $\varphi$ and $\psi$ share a propositional variable" in the SRP and WRP in [1] and [7] (see [22]).

\section{References}

[1] Anderson, A. R., and N. D. Belnap, Jr., "The pure calculus of entailment," Journal of Symbolic Logic, vol. 27 (1962), pp. 19-52. Zbl 0113.00403. MR 0154795. 472, 473, 479, 480, 481, 489

[2] Anderson, A. R., and N. D. Belnap, Jr., Entailment, I: The Logic of Relevance and Necessity, Princeton University Press, Princeton, 1975. MR 0406756. 480, 481, 482, 487, 488

[3] Anderson, A. R., N. D. Belnap, Jr., and J. M. Dunn, Entailment, II: The Logic of Relevance and Necessity, Princeton University Press, Princeton, 1992. MR 1223997. 489

[4] Beall, J. C., and G. Restall, Logical Pluralism, Oxford University Press, Oxford, 2006. 489

[5] Cintula, P., "Weakly implicative (fuzzy) logics, I: Basic properties," Archive for Mathematical Logic, vol. 45 (2006), pp. 673-704. Zbl 1101.03015. MR 2252249. DOI 10.1007/s00153-006-0011-5. 471, 473, 474, 478, 479, 480, 487

[6] Czelakowski, J., Protoalgebraic Logics, vol. 10 of Trends in LogicStudia Logica Library, Kluwer Academic, Dordrecht, 2001. MR 1828895. DOI 10.1007/978-94-017-2807-2. 479 
[7] Dunn, J. M., "Algebraic completeness for $R$-mingle and its extensions," Journal of Symbolic Logic, vol. 35 (1970), pp. 1-13. Zbl 0231.02024. MR 0288008. 471, 472, 473, 479, 480, 481, 482, 488, 489

[8] Dunn, J. M., "Relevance logic and entailment," pp. 117-224 in Handbook of Philosophical Logic, edited by D. Gabbay and F. Guenthner, vol. 166 of Synthese Library, D. Reidel, Dordrecht, 1986. 481, 482, 489

[9] Esteva, F., and L. Godo, "Monoidal t-norm based logic: Towards a logic for leftcontinuous t-norms," pp. 271-88 in Fuzzy Logic (Palma, 1999/Liptovský Ján, 2000), vol. 124 of Fuzzy Sets and Systems, North-Holland, Amsterdam, 2001. Zbl 0994.03017. MR 1860848. DOI 10.1016/S0165-0114(01)00098-7. 474

[10] Esteva, F., L. Godo, P. Hájek, and M. Navara, "Residuated fuzzy logics with an involutive negation," Archive for Mathematical Logic, vol. 39 (2000), pp. 103-24. Zbl 0965.03035. MR 1742377. DOI 10.1007/s001530050006. 474

[11] Galatos, N., P. Jipsen, T. Kowalski, and H. Ono, Residuated Lattices: An Algebraic Glimpse at Substructural Logics, vol. 151 of Studies in Logic and the Foundations of Mathematics, Elsevier B. V., Amsterdam, 2007. Zbl 1171.03001. MR 2531579. 472, 477, 478, 481, 488, 489

[12] Hájek, P., Metamathematics of Fuzzy Logic, vol. 4 of Trends in Logic-Studia Logica Library, Kluwer, Amsterdam, 1998. MR 1900263. DOI 10.1007/978-94-011-5300-3. 474, 478

[13] Hájek, P., "Fuzzy logics with noncommutative conjunctions," Journal of Logic and Computation, vol. 13 (2003), pp. 469-79. Zbl 1036.03018. MR 1999959. DOI 10.1093/logcom/13.4.469. 488

[14] Hájek, P., "Observations on non-commutative fuzzy logic," Soft Computing, vol. 8 (2003), pp. 38-43. Zbl 1075.03009. 488

[15] Metcalfe, G., "Uninorm based logics," Proceedings of EUROFUSE, Warsaw, Poland, 2004, 85-90. 472

[16] Metcalfe, G., and F. Montagna, "Substructural fuzzy logics," Journal of Symbolic Logic, vol. 72 (2007), pp. 834-64. Zbl 1139.03017. MR 2354903. DOI 10.2178/js1/1191333844. 472, 473, 474, 475, 478, 484, 488, 489

[17] Meyer, R. K., "Intuitionism, entailment, negation," pp. 168-98 in Truth, Syntax, and Modality (Philadelphia, 1970), edited by H. Leblanc, vol. 68 of Studies in Logic and the Foundations of Mathematics, North-Holland, Amsterdam, 1973. MR 0396227. 489

[18] Meyer, R. K., J. M. Dunn, and H. Leblanc, "Completeness of relevant quantification theories," Notre Dame Journal of Formal Logic, vol. 15 (1974), pp. 97-121. Zbl 0272.02028. MR 0337564. 476

[19] Novak, V., "On the syntactico-semantical completeness of first-order fuzzy logic, I: Syntax and semantics," Kybernetika (Prague), vol. 26 (1990), pp. 47-66; II: "Main results," pp. 134-54. Zbl 0705.03009. MR 1042231. 476

[20] Restall, G., An Introduction to Substructural Logics, Routledge, New York, 2000. Zbl 1028.03018. 472

[21] Yager, R. R., and A. Rybalov, "Uninorm aggregation operators," Fuzzy Sets and Systems, vol. 80 (1996), pp. 111-20. Zbl 0871.04007. MR 1389951. DOI 10.1016/0165-0114(95)00133-6. 472

[22] Yang, E., "R and relevance principle revisited," Journal of Philosophical Logic, vol. 42 (2013), pp. 767-82. DOI 10.1007/s10992-012-9747-1. 472, 473, 481, 482, 483, 485, $486,487,488,489$

\section{Acknowledgments}

I thank P. Cintula and the referees for their helpful comments and suggestions for improvements to this paper. 
Department of Philosophy and Institute of Critical Thinking and Writing Chonbuk National University

Jeonju 561-756

Korea

eunsyang@jbnu.ac.kr 